\title{
Should Portal Vein Resection Be Done in Patients with Locally Advanced Pancreas Cancer?
}

\author{
Mustafa OZSOY' , Ibrahim KILINC² \\ ${ }^{1}$ Department of General Surgery, Ankara City Hospital, Bilkent, Ankara, Turkey. \\ ${ }^{2}$ Department of General Surgery, Faculty of Medicine, Yıldırım Beyazıt University, Altindag, Ankara, Turkey. \\ Correspondence \\ Ibrahim KILINC \\ Department of General Surgery, Ankara City Hospital, Bilkent, Ankara, Turkey. \\ e-mail: ikilinc8083@gmail.com
}

\begin{abstract}
The main factor affecting resection in pancreatic adenocarcinoma is the involvement of the portal vein due to the anatomical location of the pancreas. This study aimed to investigate the effect of portal vein resection on postoperative morbidity and mortality.

Patients diagnosed with pancreatic cancer who underwent pancreaticoduodenectomy and portal vein resection between January 2014 and December 2018 were included in the study retrospectively. Besides, the demographic data of the patients, postoperative morbidity, and mortality rates were documented.

Seventeen patients [mean age 62.4 years; 11 (65\%) were male, and 6 (35\%) were female] were included in the study. Endoscopic retrograde cholangiopancreatography was applied to 10 patients (58\%) with cholangitis at presentation. CA $19-9$ elevation was found in all patients, and its mean was found to be $1497 \mathrm{U} / \mathrm{L}$. Total pancreaticoduodenectomy was applied to 2 (11.7\%) of 17 patients. In one patient (5.8\%), hepatic artery resection, besides portal vein resection, was performed. The mean operation time was $2350 \mathrm{~min}$, while the average blood loss was $820 \mathrm{~mL}$. The most common TNM stage was T2N1M0 with 11 patients (64\%). The pancreatic fistula was diagnosed in 3 (20\%) of 15 patients. One patient with Grade C fistula died on the 27 th postoperative day due to sepsis and multiorgan failure. Postoperative morbidity was encountered in 10 patients (58.8\%). The average hospital stay of the patients was 12.4 days.

Portal vein resection increases the pool of patients who benefit from surgery in pancreatic cancer. However, it can be performed safely with morbidity and mortality rates similar to those of conventional pancreatectomies in experienced hands.
\end{abstract}

Key words: Morbidity, mortality, pancreatic cancer, portal vein, reconstruction, resection

\section{INTRODUCTION}

Conventional pancreaticoduodenectomy is routinely performed with mortality rates less than $5 \%$ in high-volume hospitals (1). Portal vein invasion is one of the leading factors affecting surgical resection owing to the anatomical location of the pancreas. Invasion of the portal vein by a tumor is encountered in $30 \%$ of the patients, and palliative treatments are mostly performed considering them unresectable (2). The surgery is the only treatment option to prolong the survival in pancreatic cancer; it may only be achieved in these patients by performing portal vein resection and reconstruction (PVR). Whether PVR causes an increase in postoperative morbidity and mortality rates is still a topic of discussion (3). PVR requires more dissection and additional vascular surgery compared with con- ventional pancreaticoduodenectomy. However, the impact of portal vein resection on postoperative morbidity and mortality in patients with locally advanced pancreatic cancer was examined in the study.

\section{MATERIAL AND METHODS}

\section{Study Participants}

Patients diagnosed with pancreatic cancer who underwent pancreaticoduodenectomy and portal vein resection between January 2014 and December 2018 were retrospectively included in the present study. Besides defining the vascular anatomy of all patients, triphasic abdominal tomography was preoperatively performed to detect metastatic disease. General blood analysis and levels of Carbohydrate Antigen (CA) 19-9 
were documented, in addition to demographic data including age, sex, previous surgery, and albumin levels.

\section{Surgical Technique}

Two hours before the surgery, $0.1 \mathrm{mg}$ of octreotide (Sandostatin $0.1 \mathrm{mg} / \mathrm{mL}$ ) was administered subcutaneously to all patients. For embolism prophylaxis, anti-embolism stockings and under-patient warmers were provided to the patients. The central venous catheter and the epidural catheter were also placed. The operation started after the regional cleaning. All the surgical operations were performed by a surgeon experienced in hepatobiliary surgery. Operations began with a mini-laparotomy. The inverted T incision was performed in patients without peritonitis carcinomatosis and metastatic disease during the general intra-abdominal exploration. The gastrocolic omentum was opened after the Thompson retractor installation. The right colon and the small-intestine mesentery were ventilated using the Cattel-Braasch maneuver. The duodenum and pancreatic head were mobilized with the help of an extended Kocher maneuver. The superior mesenteric artery (SMA) was dissected from the proximal left renal vein and put aside. Later, hepatoduodenal ligament dissection was started. The right, left, and common hepatic arteries were dissected. The gastroduodenal artery and the right gastric artery were cut after ligation. The choledochus was cut by turning over the junction of the cystic duct. Lymph nodes around the portal vein and anterior and lateral celiac artery were dissected up to the right diaphragmatic crus. The coronary vein was preserved for the venous drainage of the stomach. Distal subtotal gastric resection was performed in all patients.

Further, SMA dissection was performed. All lymphatic and nerve plexuses in the posterior, anterior, and lateral sides of the SMA were dissected down to the level of the first jejunal artery. The first jejunal was cut after ligation. The middle colic artery was preserved until transverse colon invasion and was ligated for colon resection in the case of invasion. The superior mesenteric vein (SMV) was secured by turning back from the lateral side of the SMA. The first 20$\mathrm{cm}$ jejunal segment was resected with its meso. The pancreas was put aside and transected by turning along the junction point of the splenic vein with the
SMV. We waited for 2 min after 5000 units of heparin administration. If portal vein resection (PVR) was to be performed partially, splenic vein ligation and liver mobilization would not be necessary. Nevertheless, the splenic vein was ligated in patients requiring full-thickness resection of the portal vein, and the liver and small-intestine mesentery were completely released to perform the tension-free anastomosis. Following SMA clamping, resection was completed via performing clamping from the distal part of the portal confluence to the SMV and portal vein. After the piece was removed from the belly, PVR continued. In partial PVRs, Goretex graft was detected at the 5/0 prolene rate. If the end-to-end anastomosis was not possible during full-thickness resections, the left renal vein was used for reconstruction. Gastrointestinal reconstruction was performed on a single loop with pancreaticojejunostomy, hepaticojejunostomy, gastroenterostomy, and Braun anastomosis. Following the hemostasis process, the surgery was terminated by placing a drain in pancreaticojejunostomy and hepaticojejunostomy anastomosis lines. Example of surgery images of PVRs are presented in Figures 1-2.

\section{Postoperative Follow-Up}

All the patients were included for the intensive care follow-up after the surgery. Administration of 0.1 $\mathrm{mg} / \mathrm{mL}$ Sandostatin, which started subcutaneously 2 $\mathrm{h}$ before the surgery, continued from the postoperative zeroeth day to the fifth day, three times in a day. For deep venous embolism prophylaxis, early mobilization and postoperative sixth-hour clexane 4000 anti-Xa/0.4 mL treatment was initiated. Clexane treatment continued until the seventh day and was then replaced by aspirin in maintenance. PVR was checked by Doppler ultrasonography on the first day after the surgery. Nasogastric drainage was drawn on the first day after the surgery, and oral administration of water was started. If the amylase levels in the samples sent from the drains were less than three times the blood amylase value on the third day after the surgery, the drains were removed. All the patients whose oral administration increased according to their tolerance were discharged with their pancreatic enzyme preparations. All of them were examined in the first month after the surgery. 


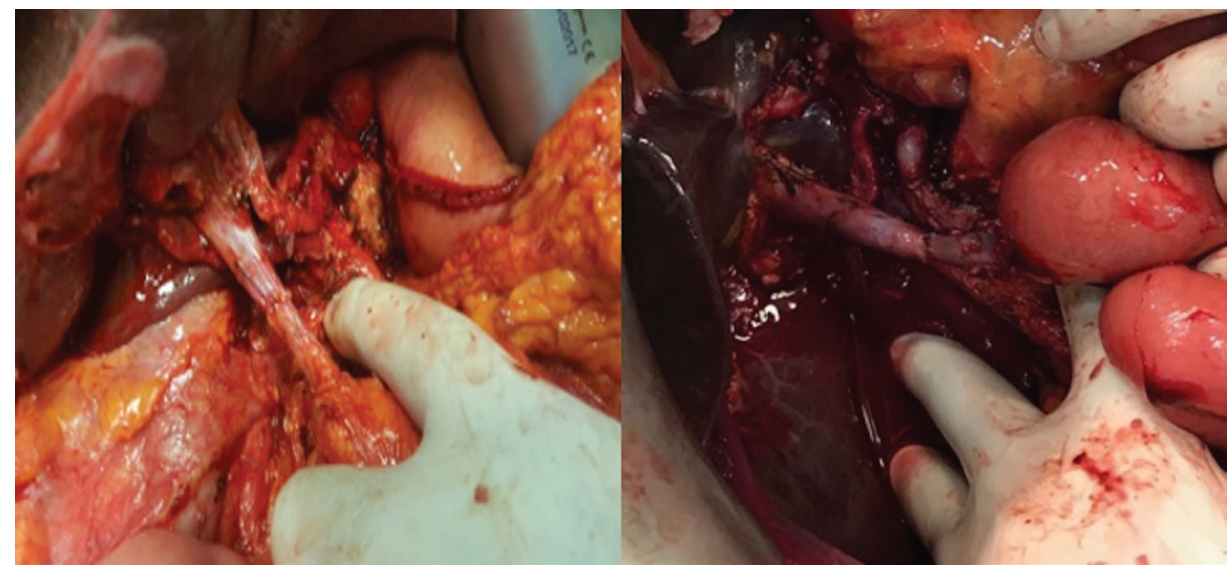

Figure 1 Examples for type 3 (segmental resection and single anastomosis) PVR cases.

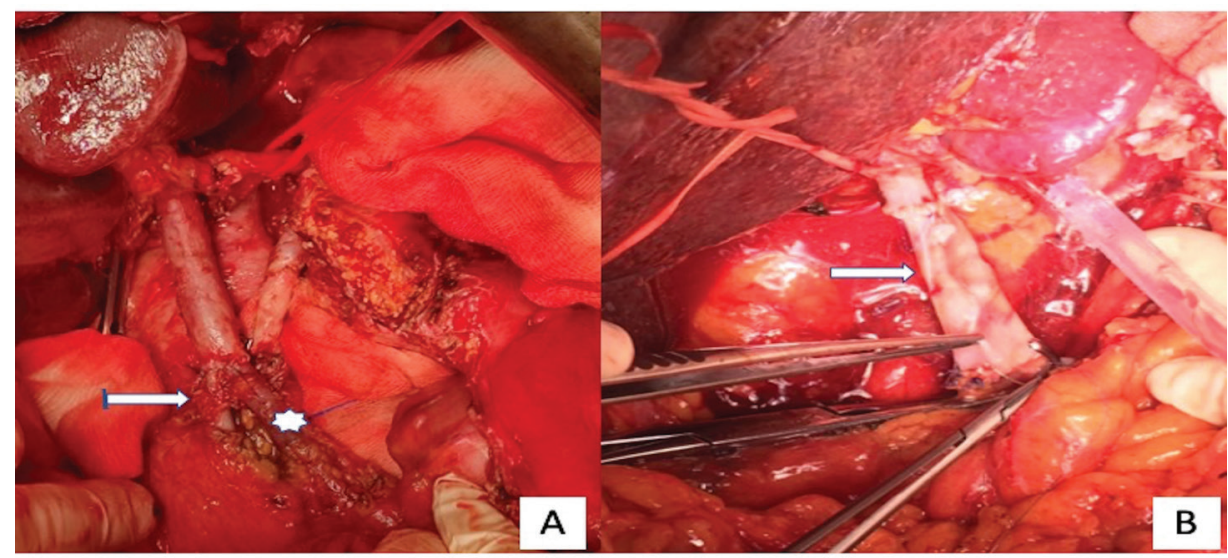

Figure 2 Type 4 (segmental resection and two anastomoses) PVR (A-) Arrow $\rightarrow$ SMV, Star $\rightarrow$ Dilated jejunal vein B-) Arrow $\rightarrow$ Autologous renal vein].

\section{Postoperative Complications}

A diagnosis of the pancreatic fistula was made in line with diagnostic criteria of the International Study Group of Pancreatic Surgery (ISGPS) in patients with a drain amylase level three times higher than the blood amylase level on the third postoperative day. When the drain bilirubin value was more than three times the blood value, the patients were diagnosed with the biliary fistula. The gastrojejunostomy anastomotic leak was checked using the methylene blue test. Sepsis was diagnosed in line with fever, clinical case, and acute-phase reactant height. In line with the histopathological examination of the surgical pieces, patients were categorized as R0, R1, or R2 based on their surgical margin positivity. Computer-controlled tomography was performed in the first postoperative month, and mortality and morbidity rates in the first month were documented.

\section{RESULTS}

Among 57 patients who underwent pancreaticoduodenectomy for pancreatic adenocarcinoma between January 2014 and December 2018, only 17 patients who underwent extended pancreaticoduodenectomy and PVR were included in our study. Patients who underwent conventional pancreaticoduodenectomy were excluded from the study. When the demographic data were examined, the average age of the patients was found to be 62.4 years. Further, of the 17 patients, 11 (65\%) were male, and 6 (35\%) 
were female. No comorbid diseases were reported in five patients (29\%). The most frequent comorbidities were chronic obstructive pulmonary disease in four patients (23\%) and diabetes in five patients (29\%). Triphasic abdominal tomography was performed in all patients during the preoperative period to detect metastatic disease and vascular anatomy. Percutaneous biopsy with interventional radiology support was performed for neoadjuvant chemotherapy in only one preoperative patient. Only one patient among the others was diagnosed with pancreatic cancer incidentally in the examinations performed for nonspecific complaints, while all patients were diagnosed with pancreatic adenocarcinoma as a result of the examinations performed for jaundice. The average of the total bilirubin levels of the patients during admissions was found to be $11.3 \mathrm{mg} / \mathrm{dL}$. A stent procedure was performed with preoperative endoscopic retrograde cholangiopancreatography (ERCP) in 10 patients (58\%) having cholangitis. CA 19-9 elevation was observed in all patients during the diagnosis. The average CA 19-9 level in male patients was $1536 \mathrm{U} / \mathrm{mL}$, while it was $1425 \mathrm{U} /$
$\mathrm{mL}$ in female patients. The average Ca19-9 level was found to be $1497 \mathrm{U} / \mathrm{mL}$. These preoperative and intraoperative results showed that pancreatic cancer was located in the head of the pancreas most frequently in nine patients (52\%), followed by uncinate localization in seven patients (41\%) (Table 1 ).

Besides the extended lymph node dissection, level 3 mesopancreatic dissection was performed in all patients having surgery due to a locally advanced pancreatic cancer. Total pancreaticoduodenectomy was performed in 2 (11.7\%) of 17 patients included in the study. Hepatic artery resection was also performed in one patient (5.8\%) besides the PVR. While PVR was partially patched with a Goretex stretch vascular graft in two patients (11.7\%), it was in the form of end-to-end anastomosis in all the other patients. In one of the patients who had end-to-end anastomosis, PVR was achieved with an autologous renal vein interposition graft. The average operation duration was $2350 \mathrm{~min}$, and the average blood loss was $820 \mathrm{~mL}$. Reproduction was observed in $7(41 \%)$ of the bile samples taken before intraoperative bile duct transection.

Table 1 Demographic and preoperative results

\begin{tabular}{|c|c|c|}
\hline \multicolumn{2}{|c|}{ PARAMETERS } & \multirow{2}{*}{$\begin{array}{l}n(\%) \\
11(65)\end{array}$} \\
\hline & Male & \\
\hline Sex & Female & $6(35)$ \\
\hline \multirow[t]{2}{*}{ Age } & Male & 60 \\
\hline & Female & 66,6 \\
\hline \multirow[t]{7}{*}{ Co-morbidity } & None & $5(29.4)$ \\
\hline & Diabetes & $5(29.4)$ \\
\hline & COPD & $4(23.5)$ \\
\hline & Hypertension & $5(29.4)$ \\
\hline & Hypertension & $2(11.7)$ \\
\hline & Chronic hepatitis & $2(11.7)$ \\
\hline & Hypothyroidism & $2(11.7)$ \\
\hline \multirow{3}{*}{ Localization } & Head & $9(52.9)$ \\
\hline & Uncinate & $7(41.1)$ \\
\hline & Head and neck & $1(5,8)$ \\
\hline \multirow[t]{2}{*}{ Total bilirubin } & Male & $11.5 \mathrm{mg} / \mathrm{dL}$ \\
\hline & Female & $11 \mathrm{mq} / \mathrm{dL}$ \\
\hline \multirow[t]{2}{*}{ CA 19-9 } & Male & $1536 \mathrm{U} / \mathrm{mL}$ \\
\hline & Female & $1425 \mathrm{U} / \mathrm{mL}$ \\
\hline \multirow[t]{2}{*}{ ERCP and Stent } & Male & $6(54.5)$ \\
\hline & Female & $4(66.6)$ \\
\hline
\end{tabular}


Escherichia coli was the most reproduced microorganism. The mean tumor diameter was $24 \mathrm{~mm}$ in the histopathological examination of the postoperative pieces; lymph node positivity was found in 15 patients (88\%). Regarding the TNM stages, the most com-monly observed stage was T2N1M0 in 11 patients (64\%). When the surgical specimens of all patients were examined, the surgical margin negativity was found to be achieved. Postopera-tive adjuvant chemoradiotherapy was not administered to two (11.7\%) patients having lymph node negativity. Adjuvant chemoradiotherapy was administered to 10 (58.8\%) of the other 12 patients. Two patients did not receive adjuvant treatment: one because of mortality and the other considering the patient's request. In 3 (20\%) of 15 patients who underwent pancreati-cojejunostomy, a diagnosis of the pancreatic fistula was made because the drain amylase level was more than 3 times the blood amylase level on the third postoperative day. The pancreatic fistula was a biochemical Grade A fistula in two of these three patients (66\%), while the Grade C fistula was observed in one patient. The patient having Grade C fistula was re-explored because of intra-abdominal bleeding on the 10th postoperative day. After the explo-ration, the gastroduodenal artery aneurysm was sutured primarily. Pancreaticojejunostomy anastomosis was spoiled, and the small intestine was transected with a stapler. The pancreatic stump was closed primarily. The patient died on the 27 th postoperative day because of sepsis and multiorgan failure. Although no other mortality (5.8\%) was observed in the present study, several postoperative morbidities were observed in 10 patients (58.8\%). One of the other two patients who had re-exploration underwent revascularization surgery on the first postoperative day after the detection of thrombus in hepatic artery resection and anastomosis. The other patient suffered a gastrojejunostomy anastomotic leak that developed on the seventh postop-erative day. No clinical problem was noted in the subsequent monitoring of these two patients. The hospitalization period of the patients was found to be 12.4 days (Table 2).

\section{DISCUSSION}

Despite current advances in pancreatic cancer, the only potentially curative treatment option is still surgery. Vascular invasion is frequently observed in a vast majority of patients because of the anatomical location of cancer during the diagnosis. Therefore, extravascular resections are required to obtain a clean surgical margin in this patient group with locally advanced stage. Although the details of extended pancreaticoduodenectomy and portal vein resection were first identified theoretically by Moore in 1951, the first pancreaticoduodenectomy with PVR was performed by Asada in Japan in $1963(4,5)$. Today, the impact of PVR on postoperative morbidity, mortality, and overall survival in pancreatic cancer is still the subject of controversy $(6,7)$. Murakami's study showed that PVR and adjuvant chemoradiotherapy gained a significant survival advantage without increasing postoperative morbidity and mortality (8). However, in the same journal, F. Giovinazzo published quite opposite results after a short time (9). In the studies conducted by Ramacciato, Carrere, and Kullmann, no difference in morbidity, mortality, and long-term survival rates was found during the early hospitalization period between standard pancreaticoduodenectomy and pancreaticoduodenectomy with PVR (10-12). Similar results were observed in two different studies by Yu XZ and Zhou Y $(6,13)$. The common view shared by the authors is that PVR can be performed routinely in high-volume hepatobiliary surgery centers without increasing postoperative morbidity and mortality. The most significant contribution of PVR in pancreatic cancer is that it increases the number of patients benefiting from the surgery. The wide margin also allows the negative margin to be taken. The most significant prognostic factors affecting the survival of patients having pancreatic cancer with vascular invasion are the tumornegative surgical margin and the depth of tumor invasion in the resected portal vein segment $(14,15)$. Another criterion for surgical success is proper patient selection. The need for vascular resection should be met with preoperative imaging methods, and surgical 
Table 2 Operative data and postoperative complications

\begin{tabular}{|c|c|c|}
\hline PARAMETERS & $\mathrm{n}(\%)$ & \\
\hline \multirow[t]{3}{*}{ Reconstruction type of portal vein } & Partial patch with Goretex graft & $2(11.7)$ \\
\hline & End-to-end anastomosis & $14(82.3)$ \\
\hline & Renal vein interposition graft & $1(5.8)$ \\
\hline Operation time & \multicolumn{2}{|c|}{$2350 \mathrm{~min}$} \\
\hline Blood loss & \multicolumn{2}{|c|}{$820 \mathrm{~mL}$} \\
\hline \multirow[t]{2}{*}{ Lymph node } & Present & $15(88)$ \\
\hline & None & $2(11.7)$ \\
\hline Tumor diameter & \multicolumn{2}{|c|}{$24 \mathrm{~mm}$} \\
\hline \multirow{5}{*}{ TNM Stage } & T1NOMO & $2(11.7)$ \\
\hline & T1N1MO & $2(11.7)$ \\
\hline & T2N1MO & $11(64.7)$ \\
\hline & T3N1M0 & $1(5.8)$ \\
\hline & T4N2MO & $1(5.8)$ \\
\hline \multirow{9}{*}{ Morbidity } & None & $7(41.1)$ \\
\hline & Atelectasis & $2(11.7)$ \\
\hline & Surgical site infection & $4(23.5)$ \\
\hline & Delayed gastric emptying & $1(5.8)$ \\
\hline & $\begin{array}{l}\text { Hepatic artery thrombosis and } \\
\text { revascularization }\end{array}$ & $1(5.8)$ \\
\hline & Biliary fistula & $1(5.8)$ \\
\hline & Gastrojejunostomy leak & $1(5.8)$ \\
\hline & Pancreatic fistula & $3(17.6)$ \\
\hline & Total & 10 patients $(58.8)$ \\
\hline Mortality & \multicolumn{2}{|c|}{$1(5.8)$} \\
\hline
\end{tabular}

planning should be done properly. Computed tomography is the most preferred imaging method in terms of revealing vascular anatomy.

While male patients dominated in the present study, the elevation of CA 19-9 levels was found in all patients. In 7 out of 10 patients provided with biliary drainage in preoperative ERCP, early reproduction of Gram-negative bacteria was observed within the bile culture. In patients without drainage, no reproduction was observed in intraoperative bile cultures. Hence, using intraoperative bile culture is quite significant for the early control of possible sepsis in patients with pancreatic cancer undergoing preoperative biliary drainage. Pancreatic fistula is often responsible for the mortality secondary to the surgery in pancreatic can- cer. Although the rate of pancreatic fistula differs in the literature, it presents with a ratio of 9.9-28.5 in the series (16). In the present study, the pancreatic fistula was found in three patients, (17.6\%) and the rate of the pancreatic fistula was similar to the data in the literature. In Wang's series of 42 patients, the operative mortality was $2.4 \%$ and the morbidity was $38 \%$ (17). In Tseng's series of 110 patients, the operative mortality was $0.9 \%$, while the perioperative morbidity was $18 \%$ (18). In Ravikumar's 230 series of cases, the mortality rate was $4.6 \%$, while the morbidity rate was $66 \%$ (19). In the present study, the mortality rate was found to be $5.8 \%$, and it was quite similar to the data in the literature. On the contrary, the morbidity rate of $58.8 \%$ was higher than the literature data. Two main reasons 
account for this high level. The patient population in the present series was relatively limited, and the morbidity classification criteria were not categorized.

PVRs are divided into four different categories by the ISGPS (20). Primary closure after partial resection makes up the first group, while the partial resection and reconstruction with a patch make up the second group. Segmental PVR and primary anastomosis were defined as the third type, and the group with at least two anastomoses with segmental resection and interpositional vascular graft was defined as the fourth group. In the study by Zhang et al., recommendations were made for the reconstruction after resection (21). They reported that when the invasion was less than 90 degrees and less than $2 \mathrm{~cm}$, PVR might be performed with a primer or patch. They proposed that primary anastomosis should be preferred after the resection when the invasion was more than 90 degrees and less than $2 \mathrm{~cm}$. They reported that if the invasion was more than 90 degrees and longer than $2 \mathrm{~cm}$, it would be appropriate to choose vascular grafts for a tension-free anastomosis. Despite the technical differences in series with $P V R$, the rate of opening of the veins during their monitoring was quite high. Although no consensus existed on anticoagulation treatment in series, the frequency of venous thrombosis varied between $9 \%$ and $18 \%$ within approximately 1 year of monitoring (22-24). In the present series, the number of patients with type 2 PVR was $2(11.7 \%)$, the number of patients with type 3 PVR was 14 (82.3\%), and the number of patients with type 4 PVR was 1 (5.8\%). In the first month of follow-ups, the portal vein was observed in all patients. The limitations of this study were its retrospective nature and the low number of patients. Prospective studies are required for more accurate results.

In conclusion, although portal vein resection increases the number of patients benefiting from surgery in pancreatic cancer, PVR in pancreatic cancer may be safely performed with morbidity and mortality rates similar to those of conventional pancreatectomies. The secret of surgical success lies in proper patient selection and perioperative patient monitoring in addition to the technique.
Conflict of interest: The authors claim that there is no conflict of interest.

\section{REFERENCES}

1. Gold EB. Epidemiology of and risk factors for pancreatic cancer. Surg Clin North Am 1995; 75: 819-43.

2. Hartwig W, Hackert T, Hinz U, et al. Pancreatic cancer surgery in the new millennium: better prediction of outcome. Ann Surg. 2011;254:311-319.

3. Luketina RR, Hackert T, Buchler MW. Vascular resection in pancreatic cancer. Indian J Surg. 2015;77:381-386.

4. Moore GE, Sako Y,Thomas LB. Radical pancreatoduodenectomy with resection and reanastomosis of the superior mesenteric vein. Surgery. 1951;30:550-553.

5. Asada S, Itaya $\mathrm{H}$, Nakamura $\mathrm{K}$ et al. Radical pancreatoduodenectomy and portal vein resection: report of two successful cases with transplantation of portal vein. Arch Surg. 1963;87:609-613.

6. Zhou Y, Zhang Z, Liu Y et all. Pancreatectomy combined with superior mesenteric vein-portal vein resection for pancreatic cancer: a meta-analysis. World J Surg. 2012;36:884-91.

7. Bockhorn M, Burdelski C, Bogoevski D et al. Arterial en bloc resection for pancreatic carcinoma. Br J Surg. 2011;98:86-92.

8. Murakami Y, Satoi S, Motoi F et al. Portal or superior mesenteric vein resection in pancreatoduodenectomy for pancreatic head carcinoma. Br J Surg. 2015;102:837-46.

9. Giovinazzo F, Turri G, Katz MH, et al. Meta-analysis of benefits of portal-superior mesenteric vein resection in pancreatic resection for ductal adenocarcinoma. Br J Surg. 2016;103:179_ 91.

10. Ramacciato G, Mercantini P, Petrucciani N, et al. Does portalsuperior mesenteric vein invasion still indicate irresectability for pancreatic carcinoma? Ann Surg Oncol. 2009;16:817-825.

11. Carrere N, Sauvanet A, Goere D, etal. Pancreaticoduodenectomy with mesentericoportal vein resection for adenocarcinoma of the pancreatic head. World J Surg. 2006;30:1526-1535.

12. Kulemann B, Hoeppner J, Wittel U, et al. Perioperative and longterm outcome after standard pancreaticoduodenectomy, additional portal vein and multivisceral resection for pancreatic head cancer. J Gastrointest Surg. 2015;19:438-444.

13. YuXZ, Li J, Fu DL, et al. Benefit from synchronous portal-superior mesenteric vein resection during pancreaticoduodenec- tomy for cancer: a meta-analysis. Eur J Surg Oncol. 2014;40: 371-378.

14. Beltrame V, Gruppo M, Pedrazzoli S, et al. Mesenteric-portal vein resection during pancreatectomy for pancreatic cancer. Gastroenterol Res Pract. 2015;2015:659-730.

15. Lapshyn $H$, Bronsert $P$, Bolm L, et al. Prognostic factors after pancreatoduodenectomy with en bloc portal venous resection for pancreatic cancer. Langenbeck's Arch Surg. 2016;401:63-69.

16. Bassi C, Butturini G, Molinari E, et al. Pancreatic fistula rate after pancreatic resection. The importance of definitions. Dig Surg. 2004;21:54-9.

17. Wang WL, Ye $S$, Yan $S$, et al. Pancreaticoduodenectomy with portal vein/superior mesenteric vein resection for patients with pancreatic cancer with venous invasion. Hepatobil Pancreat Dis Int. 2015;14:429-435.

18. Tseng JF, Raut CP, Lee JE, et al. Pancreaticoduodenectomy with vascular resection: margin status and survival duration. J Gastrointest Surg. 2004;8:935-949; discussion 949-950.

19. Ravikumar R, Sabin C, Abu Hilal M, et al. Portal vein resection in borderline resectable pancreatic cancer: a United Kingdom multicenter study. J Am Coll Surg. 2014;218:401-411.

20. Bockhorn M, Uzunoglu FG, Adham M, et al. Borderline resectable pancreatic cancer: a consensus statement by the international study Group of Pancreatic Surgery (ISGPS). Surgery. 2014;155: 977-88. 
21. Zhang XM, Zhang J, Fan $\mathrm{H}$ et al. Feasibility of portal or superior mesenteric vein resection and reconstruction by allogeneic vein for pancreatic head cancer-a case-control study. BMC Gastroenterology 2018:18:49:2-8

22. Smoot RL, Christein JD, Farnell MB. Durability of portal venous reconstruction following resection during pancreaticoduodenectomy. J Gastrointest Surg. 2006;10:13711375.
23. Glebova NO, Hicks CW, Piazza KM, et al. Technical risk factors for portal vein reconstruction thrombosis in pancreatic resec- tion. J Vasc Surg. 2015;62:424-433.

24. Chandrasegaram MD, Eslick GD, Lee W, et al. Anticoagulation policy after venous resection with a pancreatectomy: a systematic review. HPB. 2014;16:691-698. 